\title{
Bifurcations in a Nonlinear Dynamical Model between Western Pacific Subtropical High Ridge Line Index and Its Summer Monsoon Impact Factors
}

\author{
Mei Hong, ${ }^{1,2}$ Ren Zhang, ${ }^{1,2}$ Longxia Qian, ${ }^{1}$ Jingjing Ge, $^{3}$ and Jian Chen ${ }^{1}$ \\ ${ }^{1}$ Research Center of Ocean Environment Numerical Simulation, Institute of Meteorology and Oceanography, \\ PLA University of Science and Technology, No. 60 Shuanglong Road, P.O. Box 003, Nanjing 211101, China \\ ${ }^{2}$ State Key Laboratory of Atmospheric Sciences and Geophysical Fluid Dynamics, Institute of Atmospheric Physics, \\ Chinese Academy of Sciences, Beijing 100019, China \\ ${ }^{3}$ Meteorology and Hydrology Center of Nanjing C, Nanjing 210014, China
}

Correspondence should be addressed to Mei Hong; flowerrainhm@126.com

Received 6 January 2014; Accepted 20 March 2014; Published 15 April 2014

Academic Editor: Prasanta K. Panigrahi

Copyright (C) 2014 Mei Hong et al. This is an open access article distributed under the Creative Commons Attribution License, which permits unrestricted use, distribution, and reproduction in any medium, provided the original work is properly cited.

The western Pacific subtropical high (WPSH) is closely related to Asian climate. Previous studies have shown that a precise dynamical model focusing on the interaction between WPSH and other summer monsoon factors has not been developed. Based on the concept of dynamical model reconstruction, this paper reconstructs a nonlinear dynamical model of subtropical high ridge line (SHRL) and summer monsoon factors from recent 20 years data. Then, using genetic algorithm (GA), model inversion and model parameter optimization are carried out. Based on the reconstructed dynamical model, dynamical characteristics of SHRL are analyzed and an aberrance mechanism is developed, in which the external forcings resulting in the WPSH anomalies are explored. Results show that the configuration and diversification of the SHRL equilibriums have better represented the abnormal activities of the SHRL in short and medium term. Change of SHRL brought by the combination of equilibriums is more complex than that brought by mutation. The mutation behavior from high-value to low-value equilibriums of the SHRL in summer corresponds with the southward drop of the SHRL. The combination behavior of the two steady equilibriums corresponds with disappearance of the "double-ridge" phenomenon of WPSH. Dynamical mechanisms of these phenomena are explained.

\section{Introduction}

The western Pacific subtropical high (WPSH) is one of the most important components of the East Asian summer monsoon (EASM) system [1]. The intensity and position of WPSH show complex seasonal evolutions and the changes in them greatly affect the occurrence of rainy season in China, including floods, droughts, and heavy rains. For example, when WPSH reaches the northernmost position, especially in summer, it significantly influences rainfall over China and Japan [1]. In recent years, repeated anomalies of subtropical high (SH) have been observed and they have resulted in frequent occurrences of severe meteorological disasters. For example, the catastrophic Yangtze River flood in August 1998 was incurred by the abnormal southward movement of WPSH; South China and Jiangnan region experienced 14 storms from May to July in 2010 as a result of the WPSH abnormal activities. These occurrences suggest that understanding the variations of WPSH is essential for estimating the monsoon climate changes over East Asia.

Owing to its dominance on the East Asian climate, WPSH has become one of the leading topics of interest in atmospheric sciences. The seasonal variation of WPSH is closely related to the onset and withdrawal of EASM. The abrupt northward movement of WPSH from winter to summer is accompanied by abrupt changes in the circulation patterns over the East Asian monsoon area [2]. The distribution of summer rainfall in China is partly due to WPSH's seasonal variations in intensity, structure, and location 
[3, 4]. H. M. Xu et al. [5] and X. L. Xu et al. [6] argued that the development and strengthening of convection over the Bay of Bengal caused the strengthening and westward extension of SHRL. Any change in WPSH would influence the Meiyu front by influencing the convergence of tropical water vapor with extratropical airflow $[7,8]$.

Over the past decades, much effort has gone into uncovering the mechanisms governing WPSH's activities. Ren and $\mathrm{Wu}[9]$ showed that the dynamical and thermal mechanism of WPSH short-term variation was closely related to the anomalies of the South Asian high pressure and the high-latitude circulation system. The relationship between the WPSH's position and monsoon disturbance in the South China Sea as well as the Indian Ocean region was diagnosed [10]. Since the activities and variations of WPSH are extremely complex [11], the mechanisms that underlie the WPSH development and maintenance are still unresolved. The aforementioned studies have mostly dealt with the diagnostic analysis and have not dynamically modeled the interaction between WPSH and EASM. This study will attempt to develop a dynamic model and analyze the mechanisms of WPSH activities.

A dynamical model of meteorological elements or weather system is identified from the time series of observation data, which can provide a feasible way to describe the physical mechanisms of the complicated weather activities and anomalies. Zhang et al. [12, 13] investigated into the reconstruction of a nonlinear dynamical forecast model of the WPSH index based on GA and achieved satisfactory results. Since the subtropical high is a complex system with many influencing factors, incorporation of a single factor restricts the model ability. Thus, a model should incorporate more influencing factors and these factors should be objectively chosen. Considering WPSH is a member of the East Asian summer monsoon system and interacts with it, other members in the monsoon system can be introduced to further refine the dynamical model.

In our study, first the index of subtropical high ridge line (SHRL) is defined as a measurement of northward jumps and southward falls of WPSH. The rest of the paper is organized as follows. We introduce the reanalysis data of the past 20 years in Section 2. We then determine three summer monsoon factors which have higher correlation with the SHRL index by the delay-dependent method in Section 3. On this basis, the dynamical model reconstructed of the SHRL index and its impact factors are developed in Section 4 . The equilibria of the inverted dynamical model are investigated and dynamical actions of the WPSH system, such as bifurcation and mutation induced by external forces, are discussed in Section 5. Finally, Section 6 summarizes the results.

\section{Research Data}

The daily reanalysis data from May to October of past 20 years from 1991 to 2010 are provided by the US National Center for Environmental Prediction (NCEP) and the National

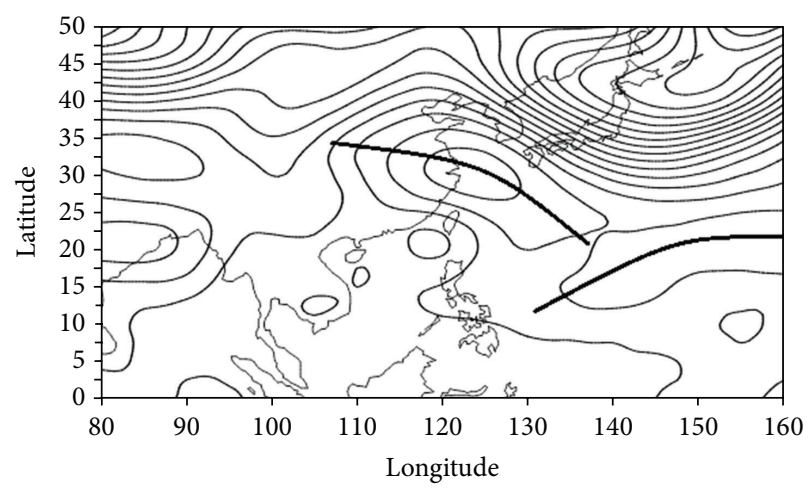

FIGURE 1: Geopotential height field at $500 \mathrm{hPa}$ on July 11, 1998 (note: heavy line represents the subtropical high ridge line).

Center for Atmospheric Research (NCAR), including (1) horizontal wind field and geopotential height field at $850 \mathrm{hPa}$ and $200 \mathrm{hPa}$; geopotential height field at $500 \mathrm{hPa}$; sea level pressure field with a resolution of $2.5^{\circ} \times 2.5^{\circ}$; (2) the sensible heat and convective precipitation rate in Gaussian grid; (3) the observed long wave radiation (OLR) of NOAA satellites.

\section{Analysis of the Impact Factors of SHRL}

3.1. Basic Facts of the Subtropical High Activities. As the dominant component of the EASM system, the WPSH activities, mainly as seasonal variations, are most frequent during the boreal summer. In general, WPSH migrates northward in a stepwise manner characterized by two distinct northward jumps. In mid-June, WPSH jumps northward for the first time, and the Meiyu season in the Yangtze River valley, Japan, and Korea begins. The second northward jump usually occurs in late July or sometimes in August. Then, WPSH shifts to its most northern position, signaling the end of the Meiyu season in the above regions and the start of the rainy season in north and northeastern China [14].

The abnormal activities of SHRL in some years often result in subtropical circulation anomalies in East Asia and extreme weather events in China [15-18], for example, "double-ridge" phenomenon of the subtropical high in 1998 (Figure 1). Thus, the SHRL position and its abnormal activities often have a great impact on the summer weather in China.

\subsection{The Delay-Dependent Analysis}

3.2.1. Correlation Analysis and Statistical Significance Test. The correlation coefficient $r_{x y}$ measures the relationship between any two variables. For the time series of two variables $x, y$ with the same sample length $n$, the correlation coefficient $r_{x y}$ can be computed from the following formula:

$$
r_{x y}=\frac{\sum_{t=1}^{n}\left(x_{t}-\bar{x}\right)\left(y_{t}-\bar{y}\right)}{\sqrt{\sum_{t=1}^{n}\left(x_{t}-\bar{x}\right)^{2}} \sqrt{\sum_{t=1}^{n}\left(y_{t}-\bar{y}\right)^{2}}} .
$$


TABLE 1: Delay-dependent analysis of the three impact factors and SHRL index.

\begin{tabular}{lcc}
\hline Number & The main member of the summer monsoon system & The correlation coefficient (time) \\
\hline 1 & The Mascarene cold high strength index (MH) & $0.91(16 \mathrm{~d})$ \\
2 & Somali low-level jet (D) & $0.90(13 \mathrm{~d})$ \\
3 & Latent heat flux of the Indian monsoon (FLH) & $0.87(4 \mathrm{~d})$ \\
\hline
\end{tabular}

Remark: in the time-delayed numbers, the positive means that the change of monsoon members is ahead of that of SHRL index and the negative indicates a lag.

The statistical significance of $r_{x y}$ or simply $r$ can be evaluated using the $t$-test. The constructed statistic $t$ is expressed as follows:

$$
t=\sqrt{n-2} \frac{r}{\sqrt{1-r^{2}}}
$$

Statistic $t$ follows the $t$-distribution with $n-2$ degrees of freedom. Given the significance level $\alpha$, we check the $t$-distribution table; if $t>t_{\alpha}$, it indicates that the two variables are significantly correlated. In fact, in the case of a fixed sample size, the critical value of the correlation coefficient $r_{c}$ can be obtained by following criterion:

$$
r_{c}=\sqrt{\frac{t_{\alpha}^{2}}{n-2+t_{\alpha}^{2}}} .
$$

If $r>r_{c}$, the significance of the $t$ test is accepted.

3.2.2. Correlation Analysis between Members of EASM System and SHRL Index. In order to further reveal correlative characteristics between members of the Asian summer monsoon system and WPSH, the index of SHRL (RI) which stands for the north and south WPSH activities is defined by the Central Meteorological Observatory [19]: in $500 \mathrm{hPa}$ geopotential height map of $2.5^{\circ} \times 2.5^{\circ}$ grids, 17 latitudes (interval $2.5^{\circ}$ ) from $110^{\circ} \mathrm{E}$ to $150^{\circ} \mathrm{E}$ are chosen and the average of the latitudes with maximum geopotential height on each latitude is calculated, the result of which is defined as RI. The larger the value of RI is, the more northward the position of SHRL is.

There are lots of members in summer monsoon system, many of which are closely related to WPSH. We use delaydependent method to analyze the correlation between these factors and RI considering the complexity. Three factors with the best correlation are filtered out for further study, which are, respectively, as follows:

(1) the Mascarene cold high strength index (MH): the average of sea level pressure grid points in the region within $\left[40-60^{\circ} \mathrm{E}, 25-35^{\circ} \mathrm{S}\right]$;

(2) Somali low-level jet (SL): the average of meridional wind at $850 \mathrm{hPa}$ in the region within $\left[40-50^{\circ} \mathrm{E}, 5 \mathrm{~S}-\right.$ $\left.5^{\circ} \mathrm{N}\right]$;

(3) latent heat flux of the Indian monsoon (FLH): the latent heat flux in the region within $\left[70-85^{\circ} \mathrm{E}\right.$, $\left.10-20^{\circ} \mathrm{N}\right]$.

Results of delay-dependent analysis between RI and the above three factors are as follows.
Table 1 shows that the correlation coefficients of the above three factors with RI have reached above 0.85 . Since the number of samples was all greater than 150, the correlation coefficient threshold was obtained using the $t$-test method. When the relevant significance level was $\alpha=0.05 r$ and degree of freedom was 120, the value of $r$ which stands for correlation coefficient threshold was 0.179 . It means that when the correlation coefficient was greater than 0.179 , the results were acceptable at the 95\% confidence level.

The Mascarene high in southern hemisphere had influence on WPSH at the early time and they showed a positive correlation, which was consistent with previous research [20]. The close relationships between Somali low-level jet (SL), latent heat flux of the Indian monsoon (FLH), and WPSH are also agreed with the previous studies $[21,22]$.

\section{Reconstruction of Dynamical Model of SHRL and Its Impact Factors}

Takens [23] strictly set forth and testified the basic idea of reconstructing dynamical system from time series of the observed data in his phase space reconstruction theory. The study suggested that evolvement of any component in the system could be determined by other components which interacted with it, and the information of components could be found in the evolvement of any other correlated component. So it is possible to reconstruct the dynamical model of system evolution using time series of observed data. The idea of dynamical system retrieval and model parameter optimization is introduced in our paper and four time series of SHRL, MH, SL, and FLH are chosen to reconstruct the dynamical model based on the above analysis of the $\mathrm{SH}$ and its impact factors in abnormal years.

4.1. Reconstruction Idea of the Dynamical Model. Suppose that the physical law of a nonlinear system evolving with time can be expressed as follows:

$$
\frac{d q_{i}}{d t}=f_{i}\left(q_{1}, q_{2}, \ldots, q_{i}, \ldots, q_{N}\right), \quad i=1,2, \ldots N
$$

where $f_{i}$ is the generalized nonlinear function of $q_{1}, q_{2}, \ldots$, $q_{i}, \ldots, q_{N}$ and $N$ is the number of state variables. $N$ can generally be determined by the complexity of dynamical 
system and measured by calculating its fractal dimensions. The difference form of (4) can be written as

$$
\begin{array}{r}
\frac{q_{i}^{(j+1) \Delta t}-q_{i}^{(j-1) \Delta t}}{2 \Delta t}=f_{i}\left(q_{1}^{j \Delta t}, q_{2}^{j \Delta t}, \ldots, q_{i}^{j \Delta t}, \ldots, q_{N}^{j \Delta t}\right) \\
j=2,3, \ldots M-1,
\end{array}
$$

where $M$ is the length of time series of observed data. The model parameters and the system structure can be gained by inversion algorithm based on the observed data. $f_{i}\left(q_{1}^{j \Delta t}, q_{2}^{j \Delta t}, \ldots, q_{i}^{j \Delta t}, \ldots, q_{N}^{j \Delta t}\right)$ is an unknown nonlinear function and we assume that $f_{i}\left(q_{1}^{j \Delta t}, q_{2}^{j \Delta t}, \ldots, q_{i}^{j \Delta t}, \ldots, q_{N}^{j \Delta t}\right)$ contains two parts: $G_{j k}$ representing the expanding items containing variable $q_{i}$ and $P_{i k}$ just representing corresponding parameters which are real numbers $(i=1,2, \ldots N, j=$ $1,2, \ldots M$, and $k=1,2, \ldots, K)$. It can be supposed that $f_{i}\left(q_{1}, q_{2}, \ldots, q_{n}\right)=\sum_{k=1}^{K} G_{j k} P_{i k}(6)$. The matrix form of (6) is $D=G P$, in which

$$
\begin{gathered}
D=\left\{\begin{array}{c}
d_{1} \\
d_{2} \\
\cdots \\
d_{M}
\end{array}\right\}=\left\{\begin{array}{c}
\frac{q_{i}^{3 \Delta t}-q_{i}^{\Delta t}}{2 \Delta t} \\
\frac{q_{i}^{4 \Delta t}-q_{i}^{2 \Delta t}}{2 \Delta t} \\
\cdots \\
\frac{q_{i}^{M \Delta t}-q_{i}^{(M-2) \Delta t}}{2 \Delta t}
\end{array}\right\}, \\
G=\left\{\begin{array}{c}
G_{11}, G_{12}, \ldots, G_{1 K} \\
G_{21}, G_{22}, \ldots, G_{2, K} \\
G_{M 1}, G_{M 2}, \ldots, G_{M, K}
\end{array}\right\}, \quad P=\left\{\begin{array}{c}
P_{i 1} \\
P_{i 2} \\
\cdots \\
P_{i K}
\end{array}\right\} .
\end{gathered}
$$

Coefficients of the above generalized unknown equation can be identified through inverting the observed data. Given a vector $D$, the vector $P$ can be solved to satisfy the above equation. It is a nonlinear system with respect to $q$; however it is a linear system with respect to $P$ (assume $P$ is unknown). So the classical least-square method can be introduced to estimate the equation and the regular equation $G^{T} G P=G^{T} D$ can be derived by making the residual sum of squares $S=$ $(D-G P)^{T}(D-G P)$ minimum.

As $G^{T} G$ is usually a singular matrix, its eigenvalues and eigenvectors can be solved easily. After removing those with 0 value, the remaining components are $K$ numbers of $\lambda_{1}, \lambda_{2}, \ldots, \lambda_{i}$ which can make up a diagonal matrix $\Lambda_{k}$ and the corresponding $K$ numbers eigenvectors which can form the diagnostic matrix $U_{L}$.

With $V_{L}=G U_{i} / \lambda_{i}$ and $H=U_{L} \Lambda^{-1} V_{L}^{T}$, equation $P=H D$ can be solved, and the parameter $P$ can be obtained.

Based on the above approach, coefficients of the nonlinear dynamical systems can be determined and the nonlinear dynamical equations of observed data can be established.

\subsection{Dynamical Model Retrieval of SHRL and Its Impact} Factors Based on the Search of Genetic Algorithm (GA). The existing parameter estimation methods such as neighborhood searching method and least-square estimation are mostly one-way search which needs to travel the entire parameter space, so the searching efficiency is low. Due to the limitation of the error gradient convergence and its dependence on the initial solution, parameter estimation is prone to fall into local optimum, rather than global optimum [24]. GA is a method extensively used as a global optimization which has been developed in recent years. GA has excellence in global searching and parallel computing and error convergence rate can been improved obviously by GA, so it is very helpful in optimizing parameters [25]. Therefore, we introduce GA to reconstruct dynamical models and optimize model parameters based on the above four time series of SHRL, FLH MH, and $D$ represented by $T_{1}, T_{2}, T_{3}$, and $T_{4}$.

With the minimum square error $S=(D-G P)^{T}(D-G P)$ as the bound, the way of the model parameter inversion follows the basic principle in Section 4.1 and seeks population (solutions) and parallel mode to search for the optimal parameters in the parameter space.

As we know, there are a lot of two-order linear equations in geophysical fluid dynamics. A lot of equations which contain linear and quadratic terms can basically perform the main characteristic in the atmosphere and ocean, such as the famous Navier-Stokes (N-S) equation. So we suppose that the following nonlinear second-order ordinary differential equations (7) are taken as the dynamical model of SHRL and its impact factors for the retrieval and reconstruction:

$$
\begin{aligned}
\frac{d T_{1}}{d t}= & a_{1} T_{1}+a_{2} T_{2}+a_{3} T_{3}+a_{4} T_{4}+a_{5} T_{1}^{2}+a_{6} T_{2}^{2}+a_{7} T_{3}^{2} \\
& +a_{8} T_{4}^{2}+a_{9} T_{1} T_{2}+a_{10} T_{1} T_{3}+a_{11} T_{1} T_{4} \\
& +a_{12} T_{2} T_{3}+a_{13} T_{2} T_{4}+a_{14} T_{3} T_{4}, \\
\frac{d T_{2}}{d t}= & b_{1} T_{1}+b_{2} T_{2}+b_{3} T_{3}+b_{4} T_{4}+b_{5} T_{1}^{2}+b_{6} T_{2}^{2}+b_{7} T_{3}^{2} \\
& +b_{8} T_{4}^{2}+b_{9} T_{1} T_{2}+b_{10} T_{1} T_{3}+b_{11} T_{1} T_{4}+b_{12} T_{2} T_{3} \\
& +b_{13} T_{2} T_{4}+b_{14} T_{3} T_{4}, \\
\frac{d T_{3}}{d t}= & c_{1} T_{1}+c_{2} T_{2}+c_{3} T_{3}+c_{4} T_{4}+c_{5} T_{1}^{2}+c_{6} T_{2}^{2}+c_{7} T_{3}^{2} \\
& +c_{8} T_{4}^{2}+c_{9} T_{1} T_{2}+c_{10} T_{1} T_{3}+c_{11} T_{1} T_{4}+c_{12} T_{2} T_{3} \\
& +c_{13} T_{2} T_{4}+c_{14} T_{3} T_{4}, \\
\frac{d T_{4}}{d t}= & d_{1} T_{1}+d_{2} T_{2}+d_{3} T_{3}+d_{4} T_{4}+d_{5} T_{1}^{2}+d_{6} T_{2}^{2}+d_{7} T_{3}^{2} \\
& +d_{8} T_{4}^{2}+d_{9} T_{1} T_{2}+d_{10} T_{1} T_{3}+d_{11} T_{1} T_{4}+d_{12} T_{2} T_{3} \\
& +d_{13} T_{2} T_{4}+d_{14} T_{3} T_{4} .
\end{aligned}
$$

Suppose that parameter matrix $P=\left[a_{1}, a_{2}, \ldots a_{9} ; b_{1}, b_{2}\right.$, $\left.\ldots b_{9} ; c_{1}, c_{2}, \ldots c_{9}\right]$ of the above equations is the population. The objective function is the minimal residual sum of squares $S=(D-G P)^{T}(D-G P)$. The individual fitness value is $l_{i}=1 / S$ and the total fitness value is $L=\sum_{i=1}^{n} l_{i}$. In which $D$ and $G$ comes from 


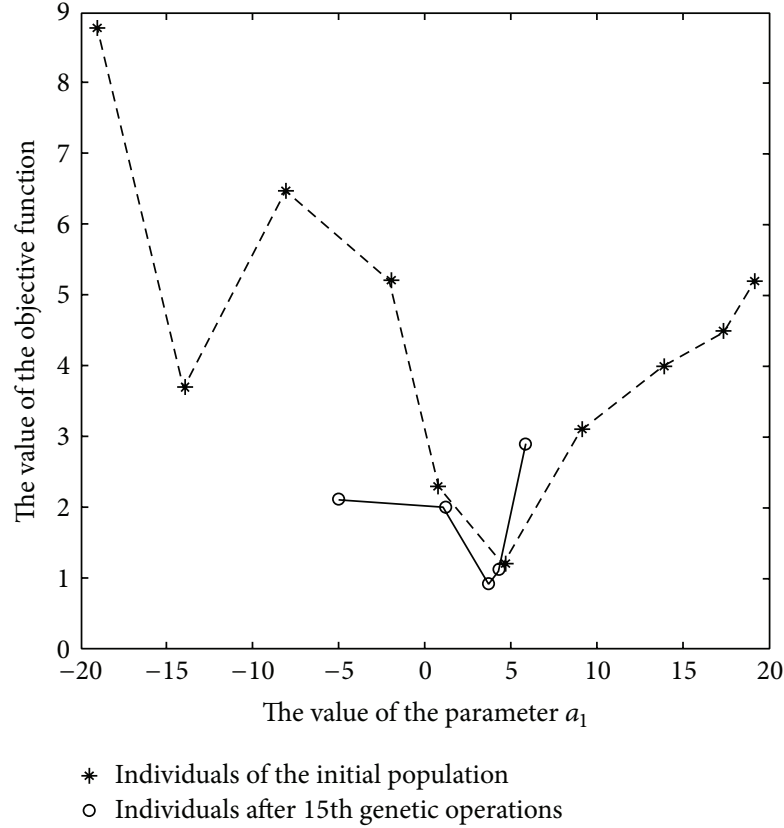

FIGURE 2: Individuals distribution of the initial population and optimization individuals after 15 th genetic operations of $a_{1}$.

the actual meteorological data: the averaged time series of SHRL from May 1 to October 31 of recent 20 years are chosen; the averaged time series of FLH from April 27 to October 27 of recent 20 years are chosen; April 15 to October 15 for $\mathrm{MH}$ and April 18 to October 18 for SL of recent 20 years are chosen. The total length for the four time series is 184 days. They are used to optimize and retrieve model parameters.

The steps of GA include coding and creation of the initial population; calculation of fitness; the male individual choice; crossover and variation. The calculation theory and detailed explanation can be seen in the related reference [25]. In the calculation, the step length is one day. Based on the above steps, taking $a_{1}$, for example, individuals distribution of the initial population and optimization individuals after 15th genetic operations of $a_{1}$ are shown in Figure 2. From the figure, after many times of genetic operations, the optimal results can be found which is more and more close to the minimum value of the objective function. Finally, after about 24 times of the genetic operations and optimization searching, the optimal result of $a_{1}=3.22 \mathrm{can}$ be got. Other parameters are also obtained by this process.

$L_{k}=\left(G_{k} P_{k}\right)$ in (8) reflects relative contribution of each item to the system evolution. After eliminating parameters of weak items with little contribution $L_{k}$, such as $a_{4}$ and $b_{1}$, we can retrieve the nonlinear dynamical model of SHRL and its impact factors, which is as follows:

$$
\begin{aligned}
\frac{d T_{1}}{d t}= & 3.22 T_{1}+0.0048 T_{2}+13.22 T_{3}-4.78 \times 10^{-8} T_{2}^{2} \\
& +2.025 \times 10^{-4} T_{1} T_{2}-1.29 \times 10^{-4} T_{2} T_{3} \\
& +3.708 \times 10^{-6} T_{2} T_{4},
\end{aligned}
$$

$$
\begin{aligned}
\frac{d T_{2}}{d t}= & -64.8571 T_{1}+0.0781 T_{2}-106.4363 T_{3}-74.3732 T_{4} \\
& -7.6904 \times 10^{-7} T_{2}^{2}+6.3939 \times 10^{-4} T_{1} T_{2} \\
& +0.0011 T_{2} T_{3}+7.3272 \times 10^{-4} T_{2} T_{4}, \\
\frac{d T_{3}}{d t}= & -0.0024 T_{2}-3.1266 T_{3}+0.28 T_{4} \\
& +2.3846 \times 10^{-8} T_{2}^{2}-0.49 T_{4}^{2}+3.3276 \times 10^{-5} T_{2} T_{3} \\
& -1.4595 \times 10^{-5} T_{2} T_{4}, \\
\frac{d T_{4}}{d t}= & -50.3035 T_{1}-0.0155 T_{2}+141.2436 T_{3}+1000.011 T_{4} \\
& +0.2325 T_{3}^{2}+3.2534 \times 10^{-5} T_{4}^{2}-0.0976 T_{1} T_{3} \\
& +0.0032 T_{3} T_{4} .
\end{aligned}
$$

To test the accuracy and rationality of the above model, practical tests are necessary. With actual data (from the model series) selected as the initial value, we carry out integral forecast experiments in the model. The correlation coefficients of the four time series between the forecasting value and the actual value are, respectively, 0.9054, 0.8291, 0.8972, and 0.8477 . The results of integral forecast experiments are in good agreement with the observation, which means that the above inversion model can basically describe the actual weather facts.

\section{Dynamical Characteristic Analysis of SHRL Aberrance}

Item 5 contains two sections. In Section 5.1, combining our model, we discuss the discrimination theory of the equilibrium stability. Based on the discrimination theory, we can find that the equilibriums of our model with different parameters are changing. And in Section 5.2, we choose four most representational parameters $a_{1}, d_{14}, c_{8}$, and $c_{4}$. Changes of these parameters in this model can lead to instability and bifurcation of the equilibrium of the WPSH system. Finally, we link the instability and bifurcation of the equilibrium with the actual weather conditions.

5.1. The Discrimination Theory of the Equilibrium Stability. The fixed points (in equilibrium state) of the dynamical system are a set of roots of dynamical system equations, and they rarely change with time. Stability of fixed points corresponds with the stability of the dynamical system, and the trajectory near the fixed points determines characteristics of the fixed points. The fixed points which often appear can be divided into four types: crunodes, saddle point, focus, and center point. Taking the model equations of SHRL and its impact factors as an example, we calculate the singular spot and discuss the stability of the equilibrium depending on the characteristics of the eigenvalues of the Jacobian. 
The nonlinear dynamical model equation (8) of SHRL and impact factors has a generalized form as the following:

$$
\begin{aligned}
f=\frac{d T_{1}}{d t}= & a_{1} T_{1}+a_{2} T_{2}+a_{3} T_{3}+a_{4} T_{4}+a_{5} T_{1}^{2}+a_{6} T_{2}^{2}+a_{7} T_{3}^{2} \\
& +a_{8} T_{4}^{2}+a_{9} T_{1} T_{2}+a_{10} T_{1} T_{3}+a_{11} T_{1} T_{4} \\
& +a_{12} T_{2} T_{3}+a_{13} T_{2} T_{4}+a_{14} T_{3} T_{4}, \\
g=\frac{d T_{2}}{d t}= & b_{1} T_{1}+b_{2} T_{2}+b_{3} T_{3}+b_{4} T_{4}+b_{5} T_{1}^{2}+b_{6} T_{2}^{2} \\
& +b_{7} T_{3}^{2}+b_{8} T_{4}^{2}+b_{9} T_{1} T_{2}+b_{10} T_{1} T_{3}+b_{11} T_{1} T_{4} \\
& +b_{12} T_{2} T_{3}+b_{13} T_{2} T_{4}+b_{14} T_{3} T_{4}, \\
h=\frac{d T_{3}}{d t}= & c_{1} T_{1}+c_{2} T_{2}+c_{3} T_{3}+c_{4} T_{4}+c_{5} T_{1}^{2}+c_{6} T_{2}^{2} \\
& +c_{7} T_{3}^{2}+c_{8} T_{4}^{2}+c_{9} T_{1} T_{2}+c_{10} T_{1} T_{3}+c_{11} T_{1} T_{4} \\
& +c_{12} T_{2} T_{3}+c_{13} T_{2} T_{4}+c_{14} T_{3} T_{4}, \\
m=\frac{d T_{4}}{d t}= & d_{1} T_{1}+d_{2} T_{2}+d_{3} T_{3}+d_{4} T_{4}+d_{5} T_{1}^{2} \\
& +d_{6} T_{2}^{2}+d_{7} T_{3}^{2}+d_{8} T_{4}^{2}+d_{9} T_{1} T_{2}+d_{10} T_{1} T_{3} \\
& +d_{11} T_{1} T_{4}+d_{12} T_{2} T_{3}+d_{13} T_{2} T_{4}+d_{14} T_{3} T_{4} .
\end{aligned}
$$

As to large-scale weather systems such as WPSH and summer monsoon system, when they are in a relatively stable state, its time-varying item of dynamical model has small value and the left items of the equations can be seen as zero. So the fixed points can be obtained through solving the equilibrium equations of the constant coefficient differential system. Then the stability can be analyzed. Formula (10) can be called the Jacobian matrix:

$$
\begin{aligned}
& \alpha_{11}=\left(\frac{\partial f}{\partial T_{1}}\right)_{T_{1_{0}}, T_{2_{0}}, T_{3_{0}}, T_{4_{0}}}, \quad \alpha_{12}=\left(\frac{\partial f}{\partial T_{2}}\right)_{T_{1_{0}}, T_{2_{0}}, T_{3_{0}}, T_{4_{0}}}, \\
& \alpha_{13}=\left(\frac{\partial f}{\partial T_{3}}\right)_{T_{1_{0}}, T_{2_{0}}, T_{3_{0}}, T_{4_{0}}}, \quad \alpha_{14}=\left(\frac{\partial f}{\partial T_{4}}\right)_{T_{1_{0}}, T_{2_{0}}, T_{3_{0}}, T_{4_{0}}} \text {, } \\
& \alpha_{21}=\left(\frac{\partial g}{\partial T_{1}}\right)_{T_{1_{0}}, T_{20}, T_{3_{0}}, T_{4_{0}}}, \quad \alpha_{22}=\left(\frac{\partial g}{\partial T_{2}}\right)_{T_{1_{0}, T_{20}, T_{30}, T_{40}}}, \\
& \alpha_{23}=\left(\frac{\partial g}{\partial T_{3}}\right)_{T_{1_{0}}, T_{2_{0}}, T_{3_{0}}, T_{4_{0}}}, \quad \alpha_{24}=\left(\frac{\partial g}{\partial T_{4}}\right)_{T_{1_{0}}, T_{2_{0}}, T_{3_{0}}, T_{4_{0}}}, \\
& \alpha_{31}=\left(\frac{\partial h}{\partial T_{1}}\right)_{T_{1_{0}}, T_{2_{0}}, T_{3_{0}}, T_{4_{0}}}, \quad \alpha_{32}=\left(\frac{\partial h}{\partial T_{2}}\right)_{T_{1_{0}}, T_{2_{0}}, T_{3_{0}}, T_{4_{0}}}, \\
& \alpha_{33}=\left(\frac{\partial h}{\partial T_{3}}\right)_{T_{1_{0}}, T_{2_{0}}, T_{3_{0}}, T_{4_{0}}}, \quad \alpha_{34}=\left(\frac{\partial h}{\partial T_{4}}\right)_{T_{1_{0}}, T_{2_{0}}, T_{3_{0}}, T_{4_{0}}} \text {, }
\end{aligned}
$$

$$
\begin{array}{ll}
\alpha_{41}=\left(\frac{\partial m}{\partial T_{1}}\right)_{T_{1_{0}}, T_{2_{0}}, T_{3_{0}}, T_{4_{0}}}, & \alpha_{42}=\left(\frac{\partial m}{\partial T_{2}}\right)_{T_{1_{0}}, T_{2_{0}}, T_{3_{0}}, T_{4_{0}}}, \\
\alpha_{43}=\left(\frac{\partial m}{\partial T_{3}}\right)_{T_{1_{0}}, T_{2_{0}}, T_{3_{0}}, T_{4_{0}}}, & \alpha_{44}=\left(\frac{\partial m}{\partial T_{4}}\right)_{T_{1_{0}}, T_{2_{0}}, T_{3_{0}}, T_{4_{0}}} .
\end{array}
$$

The eigenvalue $\lambda$ of the Jacobian matrix of the equations satisfies the next formula:

$$
\left[\begin{array}{cccc}
\alpha_{11}-\lambda & \alpha_{12} & \alpha_{13} & \alpha_{14} \\
\alpha_{21} & \alpha_{22}-\lambda & \alpha_{23} & \alpha_{24} \\
\alpha_{31} & \alpha_{32} & \alpha_{33}-\lambda & \alpha_{34} \\
\alpha_{41} & \alpha_{42} & \alpha_{43} & \alpha_{44}-\lambda
\end{array}\right]=0
$$

The equation of the eigenvalues can be converted as follows:

$$
\begin{aligned}
& \left(\alpha_{11}-\lambda\right)\left(\alpha_{22}-\lambda\right)\left(\alpha_{33}-\lambda\right)\left(\alpha_{44}-\lambda\right)+\alpha_{12} \alpha_{23} \alpha_{34} \alpha_{41} \\
& +\alpha_{13} \alpha_{24} \alpha_{31} \alpha_{42}+\alpha_{14} \alpha_{21} \alpha_{32} \alpha_{43}-\alpha_{41} \alpha_{32} \alpha_{23} \alpha_{14} \\
& -\alpha_{24}\left(\alpha_{33}-\lambda\right) \alpha_{42}\left(\alpha_{11}-\lambda\right)-\alpha_{34} \alpha_{43} \alpha_{12} \alpha_{21} \\
& -\alpha_{13}\left(\alpha_{44}-\lambda\right) \alpha_{31}\left(\alpha_{22}-\lambda\right)=0 .
\end{aligned}
$$

So solutions of this four-order equation can be gotten, which are the eigenvalues of $\operatorname{DF}\left(x^{*}\right)$. We can judge whether the equilibrium is stable at this time according to the following three theorems.

Theorem 1. When all of the eigenvalues of $D F\left(x^{*}\right)$ have negative real part, the equilibrium of system (9) is asymptotically stable.

Theorem 2. When at least one of the eigenvalues of $D F\left(x^{*}\right)$ has positive real part, the equilibrium of system (9) is unstable under Lyapunov sense.

Theorem 3. When all of the eigenvalues of $D F\left(x^{*}\right)$ have zero real part, the stability of the equilibrium in system (9) depends on the higher-order terms of the Taylor series.

The situation of Theorem 3 is called critical case, which often appears in the conservative system. Based on the above discussion, we can easily judge the stability of the equilibrium states.

5.2. Mechanisms of the Dynamical Characteristics of SHRL Aberrance. Based on the above equilibrium theory, we try to discuss and explain the abnormal mechanism of SHRL from the dynamical view of the model. We mainly investigate the abnormal changes of time series.

With time-varying items (the left items) of (8) seen as zero, the dynamical equilibrium equations which describe SHRL activity can be gotten. Then we change one parameter value of the equations by numerical calculation and the distribution of the equilibrium also changes, which can approximately reflect evolvement of SHRL system with external parameters. 


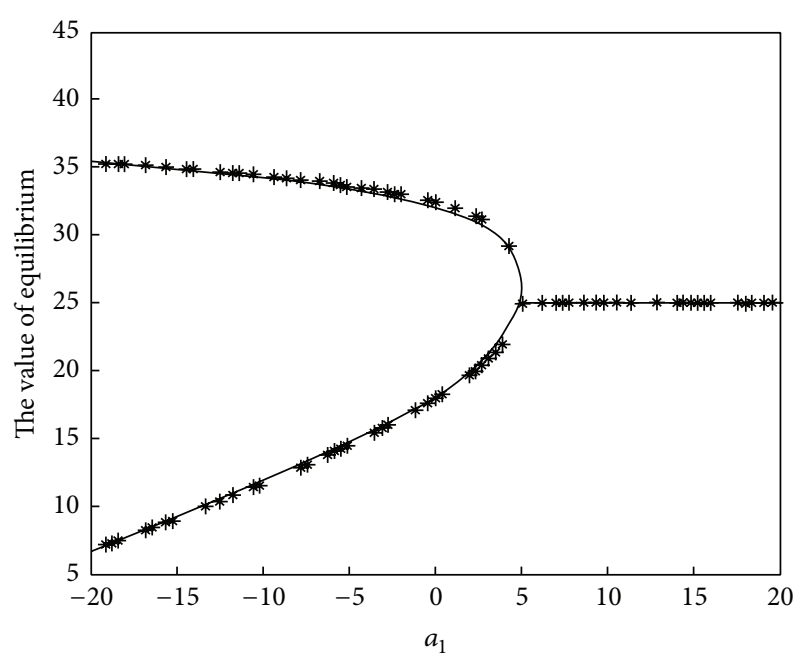

* The stable equilibrium

FIgURE 3: The stable equilibriums of the SHRL varying with $a_{1}$.

If the number and the style of the equilibrium are changing with different external parameters, we call this change of equilibrium as the bifurcation in the system; if there is a jumping phenomenon from a stable equilibrium to another with the change of parameters, we call that as the mutation in the system.

As there are numerous parameters in the equations, we choose four representatives to discuss the influence of different parameters on SHRL equilibriums. Actually, we have estimated the equilibrium stability of all the 29 parameters in (8), and after comparison, we find that the $a_{1}, d_{14}, c_{8}$, and $c_{4}$ are the most representational ones for the combination, bifurcation, and mutation. So parameters $a_{1}, d_{14}, c_{8}$, and $c_{4}$ for (8) are selected as representatives for discussion.

\subsubsection{Bifurcation and Combination of the Equilibrium Induced} by External Parameter $a_{1}$ and Aberrance of SHRL. The equilibrium of SHRL from the model is changing with external parameter $a_{1}$, shown in Figure 3.

Figure 3 shows how the equilibrium of the SHRL changes along with the increase of parameter $a_{1}$. When $a_{1}<$ 5.1, there are two equilibriums with very different values, which are, respectively, high value equilibrium and low value equilibrium. With the increase of $a_{1}$, the difference between the two equilibriums reduces significantly. When $a_{1}>5.1$, the two equilibriums merge into one stable equilibrium. Then with the increase of $a_{1}$, the equilibrium is stable and rarely varies. The changing process of the equilibrium in Figure 3 reflects the change of the actual weather conditions. Two stable equilibriums just report two corresponding centers of SHRL, which is obviously a "double-ridge" phenomenon.

After combination, there is only one stable equilibrium and the corresponding center of SHRL is only one which is in general situation. Facts reveal that when "double-ridge" phenomenon occurs in Western Pacific, neonatal south side of the ridge locates near $10^{\circ} \mathrm{N}$, while the north side of the ridge locates at north of $25^{\circ} \mathrm{N}$. As time goes on, the north side of

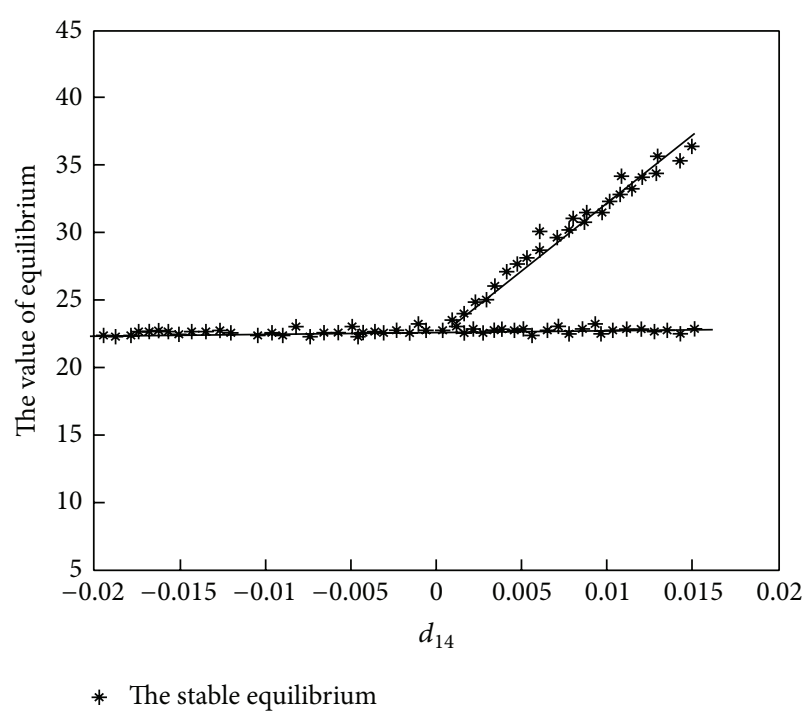

FIGURE 4: The stable equilibriums of the SHRL varying with $d_{14}$.

the ridge weakens and vanishes, while the south side of the ridge is maintained and developed. So the "reconstruction" of SHRL in the south side is completed and the second plum rain period may appear in the Yangtze River Basin. This indicates that both equilibriums of SHRL (north side and south side) can coexist with the right impact of external forcings or physical factors, which can well explain "doubleridge" phenomenon of WPSH in 1998 summer (Figure 1). Studies show that WPSH has undergone unusual changes and there was a second plum rain in the middle and lower reaches of Yangtze River [9]. In rainy interval which started after the first plum rain period and ended before the second one, "double-ridge" phenomenon of WPSH occurred [26]. The "double-ridge" phenomenon which runs through the "reconstruction" process of WPSH significantly signals the discontinuous southward retreat of SHRL and is greatly beneficial for flood forecast in the Huaihe River basin. All show that the parameter $a_{1}$ is an important external forcing which may cause a stable single ridge of WPSH to become the abnormal double ridges.

\subsubsection{Bifurcation and Discretization of the Equilibrium} Induced by External Parameter $d_{14}$ and Aberrance of SHRL. The equilibrium of SHRL from the model of SHRL and its impact factors is changing with external parameter $d_{14}$, shown in Figure 4.

Figure 4 shows how the equilibrium of the SHRL changes along with the increase of parameter $d_{14}$. When $d_{14}<$ 0.001 , there is one equilibrium state. But when $d_{14}>$ 0.001 , the equilibrium is divided into two equilibriums. With the increase of $d_{14}$, the low value equilibrium maintains stability. Figure 4 reports the dynamical characteristics of SHRL model in summer. Combined with the weather facts, before bifurcation, there is a single stable equilibrium and the corresponding WPSH range (5900 potential line) is located at low latitude (center $20^{\circ} \mathrm{N}$ ) in the Western Pacific ocean, which is similar to the circulation in early spring season; after 


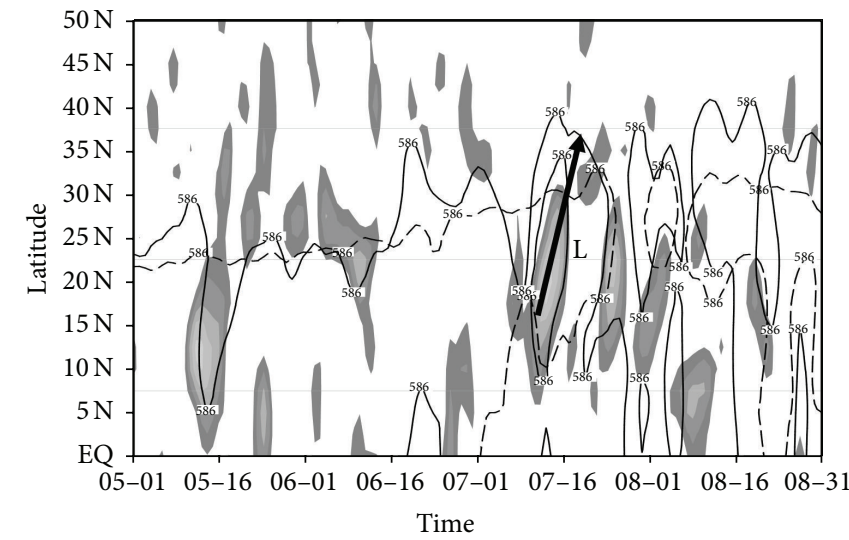

(a)

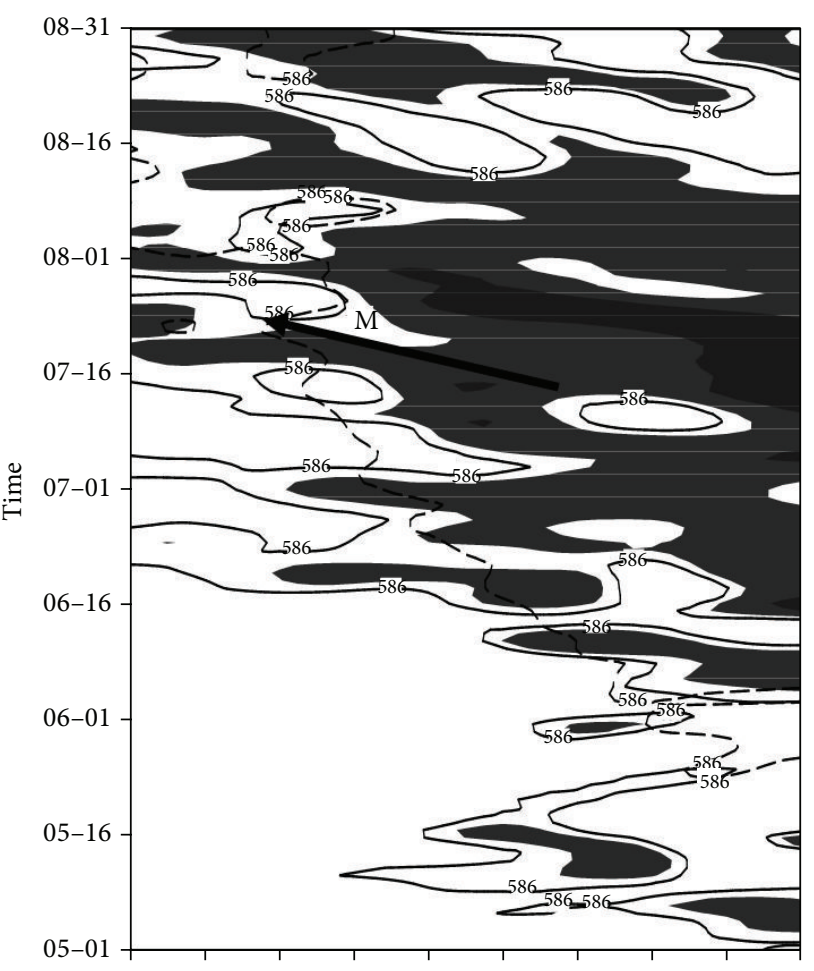

$90 \mathrm{E} \quad 100 \mathrm{E} 110 \mathrm{E} 120 \mathrm{E} 130 \mathrm{E} 140 \mathrm{E} 150 \mathrm{E} 160 \mathrm{E} 170 \mathrm{E} 180 \mathrm{E}$ Longitude

(b)

FIgURE 5: From May to August 2006 (a) latitude-time distribution of 586 dagpm contour along $110^{\circ} \mathrm{E} \sim 120^{\circ} \mathrm{E}$ averaged; (b) longitude-time distribution of $500 \mathrm{hPa}$ geopotential height along $27.5^{\circ} \mathrm{N} 33.5^{\circ} \mathrm{N}$ averaged (unit: dagpm) (note: in (a), shaded areas represent the latitudetime distribution of OLR anomaly $\leq-20 \mathrm{~W} \cdot \mathrm{m}^{-2}$, while in (b), deep shaded areas indicate that potential height is greater than 586 and 588 dagpm region. The long-dashed line is the multiyear average of 586 dagpm line in both figures).

bifurcation, there appears two possible patterns of the WPSH activities: one is that westward extended of WPSH to the South China with the other part still remaining at sea, while the northward process of WPSH is not obvious which also remains at low equilibrium state after bifurcation; the other is expressed as significant enhancement and northward lifting of WPSH (5900 centre locates near $30^{\circ} \mathrm{N}$ and will extend to about $40^{\circ} \mathrm{N}$ ). The process is similar to abnormal strengthening of WPSH and northward or westward circumfluence in summer of 2010 (the arrow L and M in Figure 5), resulting in 14 storms from May to July in South China and Jiangnan areas and 10 storms from mid-July to early September in northern and western regions. All of these indicate that the parameter $d_{14}$ is an important external forcing which may cause abnormal enhancement and northward jump of WPSH.

\subsubsection{Bifurcation and Mutation of the Equilibrium Induced by} External Parameters $c_{8}, c_{4}$ and Aberrance of SHRL. The stable equilibriums of the SHRL change along with $c_{8}, c_{4}$, and the comparison is shown in Figure 6.

Figure 6 displays that changes of equilibriums in the above two figures are similar, both showing bifurcation and mutation. In Figure 6(a), the stable equilibriums change with $c_{8}$. When $c_{8}<-1.9, \mathrm{SHRL}$ is in a low value equilibrium ranging from 10 to 15 . When $c_{8}>-1.9$, the equilibrium of SHRL suddenly jumps to higher value states between 22 and 28. When $c_{8}>1.1$, the equilibrium again suddenly leaps and locates in high value state of 35-40. What Figure 6(a) reflects on the weather situation is two northward hopping processes of WPSH. In early June, SHLR first jumps north over $20^{\circ} \mathrm{N}$, and the plum rain in the Yangtze River and Huaihe River basin starts; at the beginning of July, SHLR jumps north again over $25^{\circ} \mathrm{N}$ when the rain belt moves from Jianghuai areas to North China. This weather situation is universal and appears in most years. All this shows that parameter $c_{8}$ is an external forcing which leads to normal northward jump of WPSH.

The changing of equilibrium with external parameters in Figure 6(b) is very similar to that in Figure 6(a), but there is only one jumping process in Figure 6(b), while there are two in Figure 6(a). In Figure 6(b), the equilibrium value suddenly jumps from 15 to 35 when $c_{4}>0.54$, which represents a strong northward hopping process of WPSH with SHRL changing from $15^{\circ} \mathrm{N}$ to $35^{\circ} \mathrm{N}$. The impact on actual weather conditions is that WPSH jumps directly to the Yellow River Basin without stopping over at the Yangtze River basin which results in the "empty plum" phenomenon. For example, in June 2006, with the second westward extending and northward jumping 


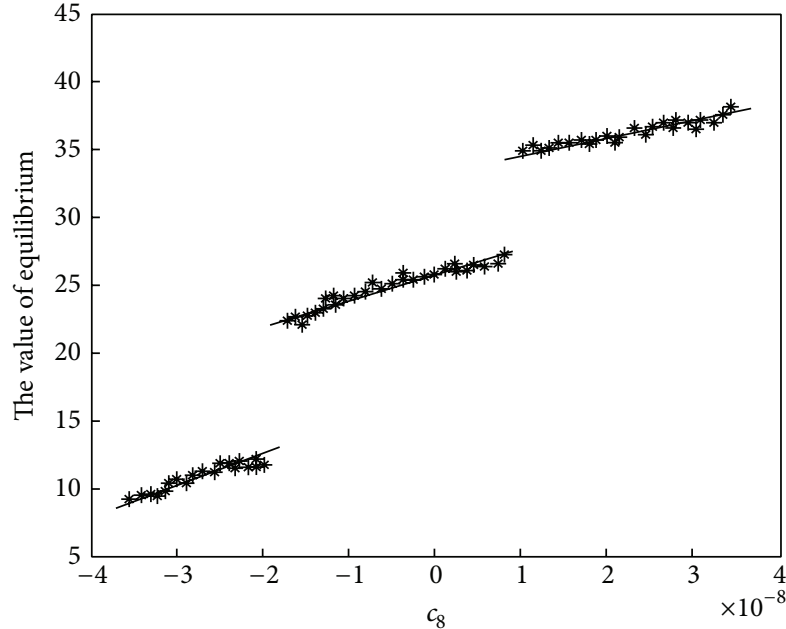

* The stable equilibrium

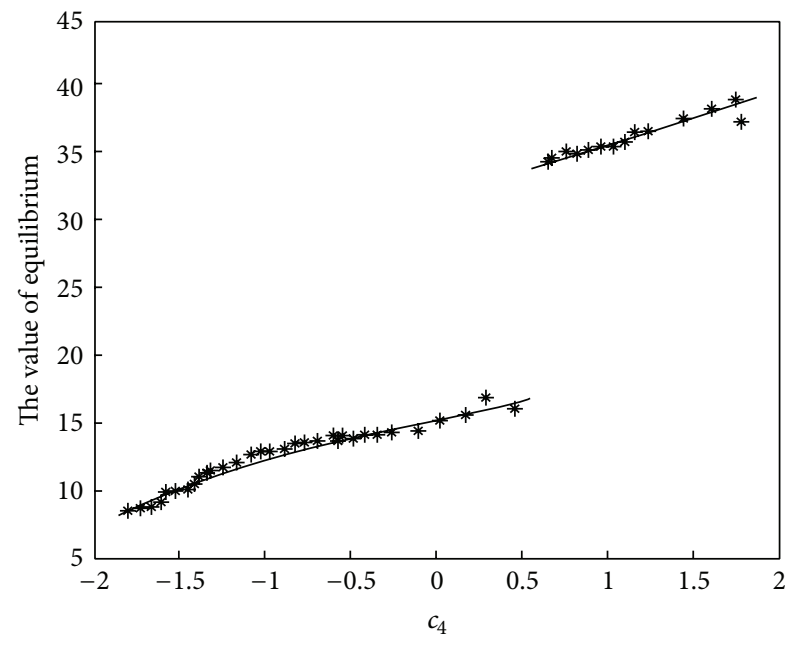

* The stable equilibrium

(a)

(b)

FIgURE 6: The stable equilibrium of the SHRL varying with $c_{8}$ (a) and $c_{4}(\mathrm{~b})$.

of WPSH, SHRL quickly moved to $35^{\circ} \mathrm{N}$, which led to the second continuous empty plum in the middle and lower reaches of the Yangtze River since 2005. Rain belt appeared in the Huaihe River Basin and WPSH was very unstable which oscillated near $30^{\circ} \mathrm{N}$. Above analysis indicates that external parameters $c_{4}$ and $c_{8}$ maybe the external forcing leading to WPSH northward jump.

\section{Summary}

The WPSH is an important member of the East Asian summer monsoon system, which is in a nonlinear system together with the monsoon circulation. The WPSH also plays a major role in East Asian climate. Previous studies focused on uncovering the laws of its abnormal activities and dynamic mechanism. In early stages the low-order spectral method obtained multiequilibrium states were often utilized for the high's northward displacement and subsequently, Fan and Miao [27] explained the genesis by means of Rossby soliton theory. However, WPSH differs in formation more greatly from blocking highs, and particularly the meridional shift of the high is just a change in position, with few or no change for the related flow pattern, which differs greatly from the multiple equilibria of different space flow patterns associated with meridional and zonal circulations of high and low index at extratropics. Therefore, it seems difficult to expound the variation of WPSH by use of the multiequilibrium theory and the result differs to greater extent from observational fact. So far, few reports published have solved this problem. To overcome the limitations for the inconsistency of a soliton solution with the facts of the real atmosphere, we use the novel idea of reconstructing dynamical system to invert the model of SHRL and its impact factors from time series of the observational data. And the dynamical characteristics can be analyzed. The major results are summarized as follows.
(1) Three summer monsoon factors which have close relationship with the subtropical high ridge line (SHRL) index are found by delay-dependent method: Mascarene cold high intensity, Somali low-level jet, and latent heat flux of the Indian monsoon.

(2) Due to the complexity of the EASM system and summer activities of WPSH, the dynamical model to describe WPSH activities has not been established so far. In this study, we put forward a novel idea to reconstruct dynamical model of SHRL and its impact factors.

(3) We investigated the mechanism of SHRL aberrance and the equilibrium stability of the WPSH system and analyzed the dynamical characteristics and actions, such as bifurcation and mutation induced by the external forcings. Changes of parameters in this model can lead to instability and bifurcation of the equilibrium of the WPSH system.

(4) We link the developed mechanism with the actual weather conditions and further analyze characteristics of the actual WPSH and summer monsoon system activities. We find that the distribution and variation of the equilibrium of SHRL and its impact factors have good correspondence with the actual medium-short abnormal activities of the WPSH. Our analysis also shows that the change brought by equilibrium change of bifurcation and combination is more complex than that brought by mutation. In particular, the hop from low value equilibrium to the high one in the summer WPSH system corresponds with the dynamical behaviour of the WPSH northward jump. Combination of the two steady equilibriums corresponds with the disappearance of the "doubleridges" phenomenon of the WPSH. All are applied to 
characteristics representation and mechanism explanation of the empty plum rain in the middle and lower reaches of the Yangtze River in 2006 and "doubleridge" phenomenon in 1998 summer.

\section{Conflict of Interests}

The authors declare that there is no conflict of interests regarding the publication of this paper.

\section{Acknowledgments}

This study is supported by the Chinese National Natural Science Fund for young scholars (41005025/D0505), the Chinese National Natural Science Fund (41375002 and 41075045), and the Chinese National Natural Science Fund (BK2011123) of Jiangsu Province.

\section{References}

[1] S. Y. Tao and S. Y. Xu, "Circulation characteristics in association with persistent summer drought and flood in theYangtzeHuaihe River reaches," Acta Meteorologica Sinica, vol. 32, pp. 1$18,1962$.

[2] D. Ye and B. Zhu, Some Fundamental Problems of the General Circulation of the Atmosphere, Science Press, Beijing, China, 1958.

[3] J. W. Bergman and H. H. Hendon, "The impact of clouds on the seasonal cycle of radiative heating over the Pacific," Journal of the Atmospheric Sciences, vol. 57, no. 4, pp. 545-566, 2000.

[4] C. Deser and A. S. Phillips, "Simulation of the 1976/77 climate transition over the North Pacific: sensitivity to tropical forcing," Journal of Climate, vol. 19, no. 23, pp. 6170-6180, 2006.

[5] H. M. Xu, J. H. He, and B. Zhou, "The possible mechanism of the Western Pacific subtropical high northward jump and western extension and the evolution characteristics of atmospheric circulation before the Meiyu onset," Journal of Applied Meteorology, vol. 12, no. 2, pp. 150-158, 2001.

[6] X. L. Xu, H. M. Xu, and D. Si, "Relationship between June sustained torrential rain in South China and anomalous convection over the bay of Bengal," Journal of Nanjing Institute of Meteorology, vol. 30, pp. 463-471, 2007.

[7] K. Ninomiya and C. Kobayashi, "Precipitation and moisture balance of the Asian summer monsoon in 1991 part II: moisture transport and moisture balance," Journal of the Meteorological Society of Japan, vol. 77, no. 1, pp. 77-99, 1999.

[8] A.-L. Gibelin and M. Déqué, "Anthropogenic climate change over the Mediterranean region simulated by a global variable resolution model," Climate Dynamics, vol. 20, no. 4, pp. 327-339, 2003.

[9] R. C. -Ren and G. X. Wu, "On the Short-term structure and formation of the subtropical anticyclone in the summer of 1998," Acta Meteorologica Sinica, vol. 61, pp. 180-195, 2003.

[10] B. J. Hoskins and B. Wang, "Large-scale atmospheric dynamics," in The Asian Monsoon, B. Wang, Ed., pp. 357-415, Springer, 2006.

[11] K. Krishna Kumar, M. Hoerling, and B. Rajagopalan, "Advancing dynamical prediction of Indian monsoon rainfall," Geophysical Research Letters, vol. 32, no. 8, Article ID L08704, 2005.
[12] R. Zhang, M. Hong, Z.-B. Sun et al., "Non-linear dynamic model retrieval of subtropical high based on empirical orthogonal function and genetic algorithm," Applied Mathematics and Mechanics, vol. 27, no. 12, pp. 1645-1653, 2006.

[13] R. Zhang, M. Hong, H. Z. Wang, Y. D. Chen, and Y. L. Wan, "Retrieval the non-linear dynamic forecast model of El Nino/La Nina index based on the genetic algorithm optimization," Chinese Journal of Geophysics, vol. 51, pp. 633-645, 2008.

[14] J. H. He, J. H. Ju, Z. P. Wen, J. Lü, and Q. Jin, "A review of recent advances in research on Asian monsoon in China," Advances in Atmospheric Sciences, vol. 24, no. 6, pp. 972-992, 2007.

[15] S. Y. Tao, Y. Q. Ni, S. X. Zhao et al., The Study on Formation Mechanism and Forecast of the 1998 Summer Rainstorm in China, Meteorological Press, 2001.

[16] T. Miyasaka and H. Nakamura, "Structure and formation mechanisms of the northern hemisphere summertime subtropical highs," Journal of Climate, vol. 18, no. 23, pp. 5046-5065, 2005.

[17] T. Nitta, "Convection activities in the tropical western Pacific and their impact on the Northern Hemisphere circulation," Journal of the Meteorological Society of Japan, vol. 67, pp. 375383, 1987.

[18] L. T. Chen, "The role of the anomalous snow cover over the qinghai-xizang plateau and ENSO in the great floods of 1998 in the changjiang river valley," Chinese Journal of Atmospheric Sciences, vol. 25, pp. 184-192, 2001.

[19] The Central Meteorological Station forecast group, The LongRange Weather Forecast Technology Experience, The Central Meteorological Station Press, Beijing, China, 1976.

[20] F. Xue, H. J. Wang, and J. H. He, "The influence of Mask Lin high and Australia high interannual variation to the East Asian summer monsoon precipitation," Chinese Science Bulletin, vol. 3, pp. 19-27, 2003.

[21] R. Seager, R. Murtugudde, N. Naik, A. Clement, N. Gordon, and J. Miller, "Air-sea interaction and the seasonal cycle of the subtropical anticyclones," Journal of Climate, vol. 16, pp. 19481966, 2003.

[22] P. J. Webster, A. M. Moore, J. P. Loschnigg, and R. R. Leben, "Coupled ocean-atmosphere dynamics in the Indian Ocean during 1997-98," Nature, vol. 401, no. 6751, pp. 356-360, 1999.

[23] F. Takens, "Detecting strange attractors in fluid turbulence," Lecture Notes in Mathematics, vol. 898, pp. 361-381, 1981.

[24] L. Xiang, Z. Chen, and C. S. Xiao, "Discrete and dynamic optimization problems in operation management," Discrete Dynamics in Nature and Society, vol. 2013, Article ID 816382, 2 pages, 2013.

[25] J. Zhao, W. Y. Liu, and J. Wei, "Pricing and remanufacturing decisions of a decentralized fuzzy supply chain," Discrete Dynamics in Nature and Society, vol. 2013, Article ID 986704, 10 pages, 2013.

[26] R. F. Zhan, J. P. Li, and J. H. He, "Influence of the double ridges of west pacific subtropical High on the second Meiyu over the yangtze river valley during 1998," Acta Meteorologica Sinica, vol. 62, pp. 294-307, 2004.

[27] H. Fan and J. H. Miao, "Rossby soliton theory on the formation of subtropical and blocking highs. II," The Critical Layer, Acta Meteorologica Sinica, vol. 54, no. 6, pp. 722-729, 1996. 


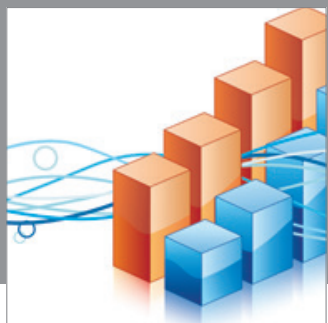

Advances in

Operations Research

mansans

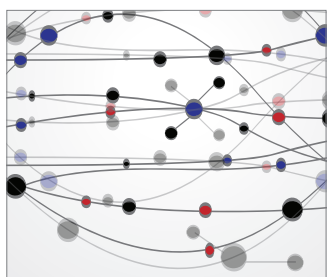

The Scientific World Journal
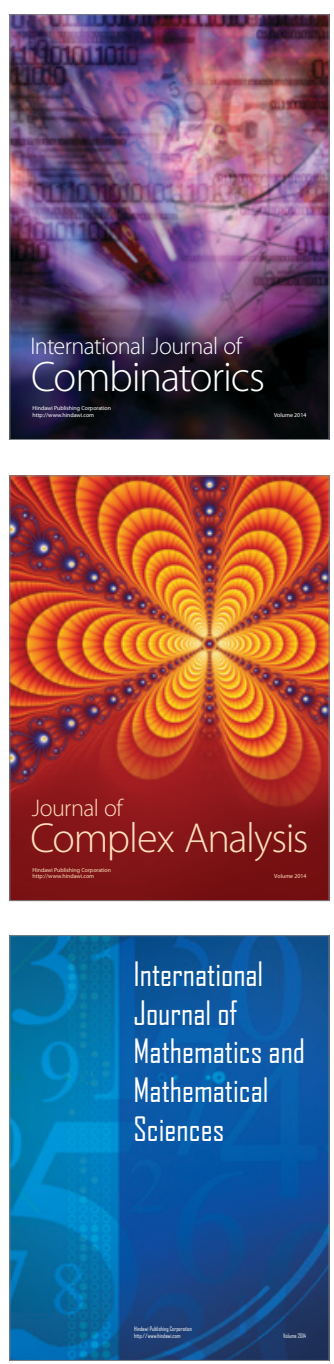
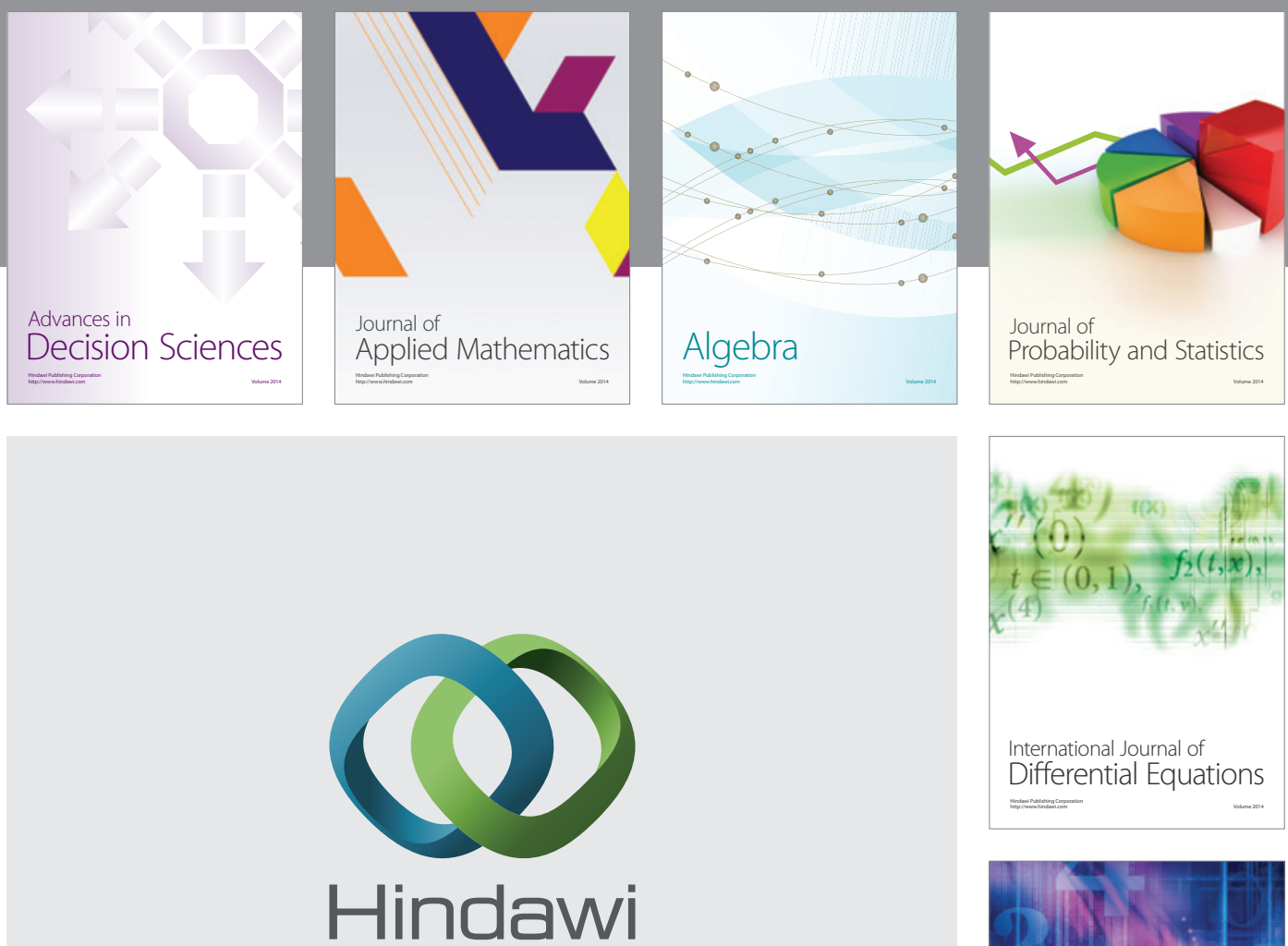

Submit your manuscripts at http://www.hindawi.com
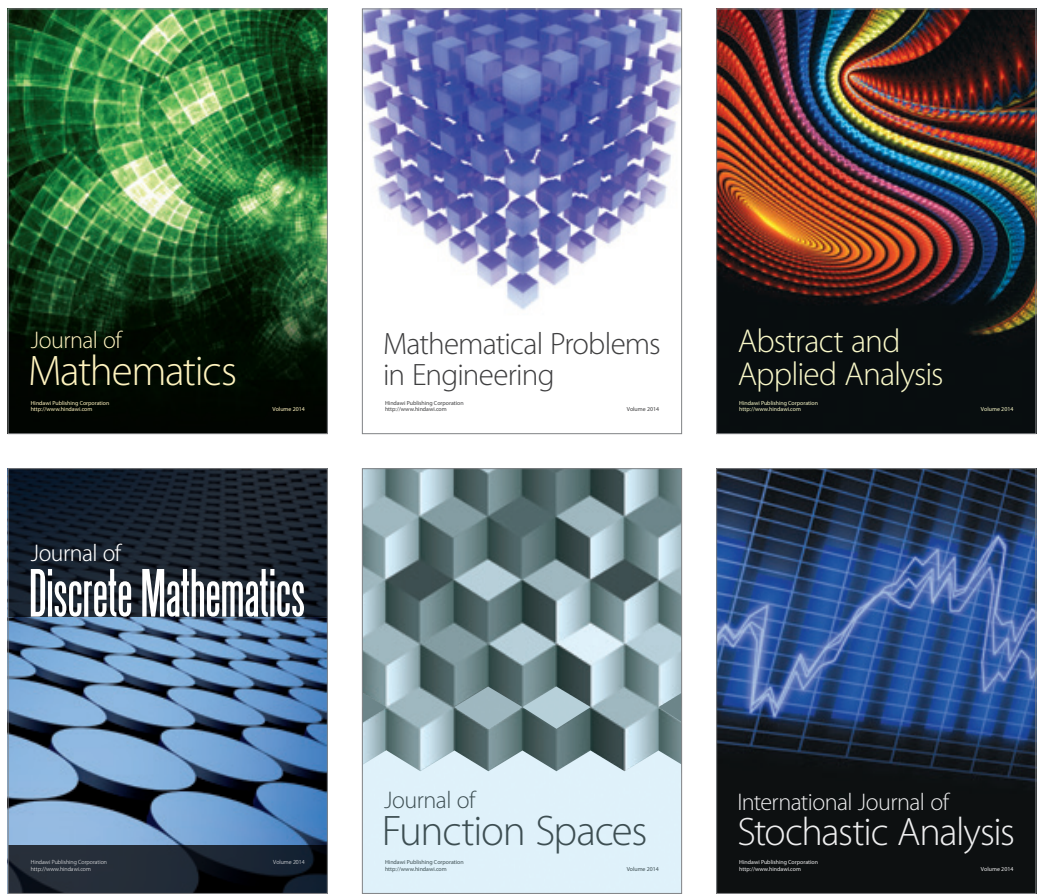

Journal of

Function Spaces

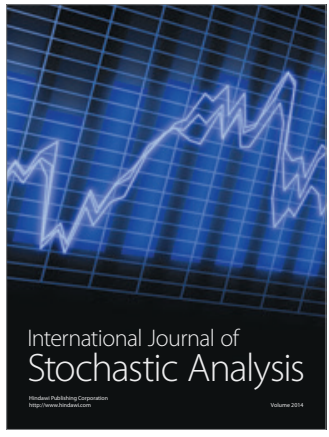

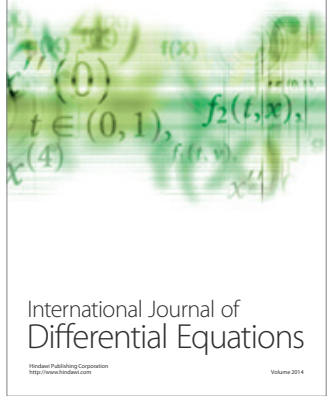
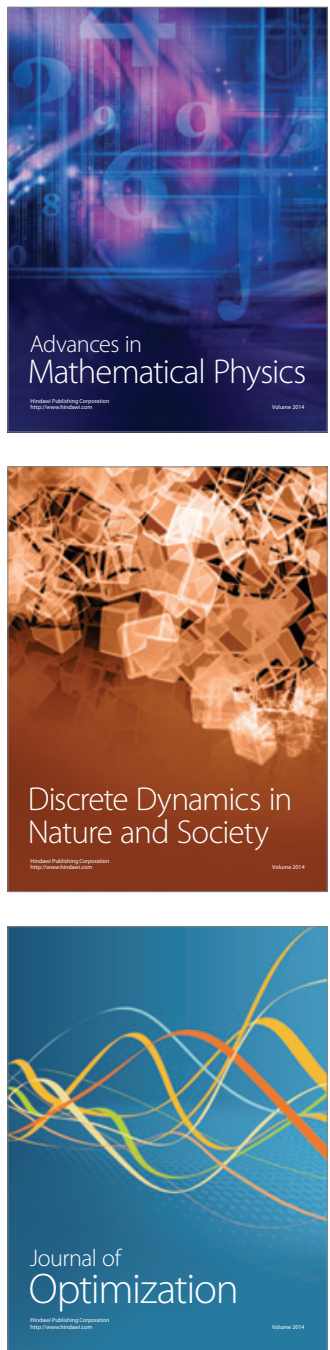